\title{
Frequency of venous thrombosis in patients with an exacerbation of chronic obstructive lung disease
}

\author{
JH WINTER, PW BUCKLER, AP BAUTISTA, FW SMITH, PF SHARP, B BENNETT, \\ AS DOUGLAS
}

From the Departments of Medicine and Nuclear Medicine, University of Aberdeen

\begin{abstract}
The presence or absence of venous thrombosis was determined in 29 patients with an acute exacerbation of chronic obstructive lung disease by the technique of autologous platelet labelling with indium-111. Deep venous thrombosis was diagnosed in 13 patients and in nine of these patients thrombosis was located proximal to the knee-that is, in areas associated with an appreciable risk of pulmonary embolism. One patient died; pulmonary emboli were found at postmortem examination. The incidence of venous thrombosis seen in this study is high enough to suggest that these patients may benefit from prophylactic antithrombotic treatment.
\end{abstract}

Pulmonary thromboembolism is a common finding at postmortem examination of patients with chronic obstructive lung disease ${ }^{2}$; the role of thromboembolism, however, in the precipitation or evolution of acute exacerbations of chronic obstructive lung disease and in the pathogenesis of the pulmonary hypertension seen in this disorder is unknown. The diagnosis of pulmonary embolism in patients with significant chronic obstructive lung disease is difficult; physical signs are usually unhelpful; lung scans may be difficult to interpret and even pulmonary angiography may fail to show small emboli. ${ }^{3}$

Although it has been suggested that pulmonary emboli in patients with chronic obstructive lung disease may originate in the right ventricle of the heart ${ }^{1}$ at least a proportion of pulmonary emboli are likely to originate from the pelvis and lower extremities, as is the case in other groups of patients. ${ }^{45}$ We have attempted to define the frequency of deep venous thrombosis in patients admitted to hospital with an exacerbation of chronic obstructive lung disease using the indium-111-labelled platelet technique. ${ }^{6}$

\section{Patients and methods}

Twenty-nine patients with an exacerbation of chronic obstructive lung disease were enrolled into Address for reprint requests: Dr J H Winter, Centre for Respirat-
ory Investigation, Glasgow Royal Infirmary, Glasgow G4 OSF.

Accepted 6 April 1983 the study. These subjects were patients admitted to the thoracic medicine wards of the Aberdeen hospitals. The patients were enrolled consecutively except during periods when the investigators were absent or when the indium labelling technique was not available.

The diagnosis of chronic obstructive lung disease was based on the following: a history of cigarette smoking, winter bronchitis, and dyspnoea progressing over several years; obstruction of the airways indicated by a ratio of forced expiratory volume in one second to forced vital capacity $\left(F E V_{1} / F V C\right)$ of less than $70 \%$; and no significant reversibility of the obstructive defect (increase of $F E V_{1}$ by more than $15 \%$ in response to inhalation of a $\beta$-agonist drug by aerosol) either during the study admission or during a previous admission to hospital. An exacerbation of chronic obstructive lung disease was defined as an increase in the severity of respiratory symptoms resulting in hospital admission. All patients received physiotherapy and treatment with controlled oxygen, antibiotic drugs, nebulised bronchodilator agents, and bed rest. Eighteen patients received treatment with corticosteroid drugs and 22 were treated with diuretic agents during their study admission. Treatment with agents affecting platelet function was avoided. Therapeutic venesection was not performed on any patient in the interval between admission to hospital and scanning after platelet labelling.

When the patients had recovered sufficiently from their exacerbation to undergo scanning, labelling of 
platelets was performed as previously described. ${ }^{6}$ During the interval between admission and scanning the patients were largely confined to bed. A full blood count, estimation of arterial blood gases (while the patient was breathing air), spirometry, and the measurement of height and weight were undertaken at this time. Scanning of the pelvis and lower limbs was performed 24 hours after platelet labelling. Body weight was determined and expressed as a percentage of average body weight on the basis of tables. ${ }^{7}$

\section{Results}

No patient had symptoms or clinical signs suggestive of deep venous thrombosis in the leg. Deep venous thrombosis was diagnosed by the indium-labelled platelet technique in 13 patients. Thrombi were confined to the calf veins in four patients and to the proximal veins (femoral, iliofemoral, and iliac veins) in two and were present in both calf and proximal veins in the remaining seven patients.

One patient, a 51-year-old woman, died whilst in hospital. Scanning showed no abnormal accumulation of platelets 16 days after her admission, when her initial exacerbation had settled. She subsequently developed a further exacerbation and died two weeks after her indium scan. Postmortem examination showed a right-sided iliofemoral vein thrombosis, pulmonary emboli, and abscesses in the right lung, which in the pathologist's opinion had formed in infarcted lung tissue. One patient in this series, a man aged 54, required artificial ventilation for $\mathbf{4 8}$ hours immediately after his admission to hospital. Indium scanning in this man nine days after admission showed bilateral calf thrombi.

The table shows the mean age, spirometric indi- ces, blood gases, percentage of average body weight, and interval between admission and scanning in patients with and without venous thrombosis. There are no significant differences between the two $\bar{D}$ groups. There was no association between the occurrence of venous thrombosis and treatment with corticosteroid or diuretic drugs.

\section{Discussion}

We showed earlier ${ }^{6}$ that the indium-labelled platelet $\stackrel{\omega}{\omega}$ technique is accurate and specific in the diagnosis of $\vec{x}$ deep venous thrombosis by comparison with ascending venography. No false positives were found in $\infty$ that study, although a few false negatives occurred, so that the technique might be expected to underes- or timate the incidence of deep venous thrombosis. The earlier study was performed on patients who $\rightarrow$ had recently had operative reduction and fixation of $\supset$ a fracture of the neck of the femur and in whom recent massive thrombosis was common; our subse- क्क quent experience with this technique in patients pre- $\overrightarrow{\vec{\theta}}$ senting with symptoms or signs suggestive of deep venous thrombosis or pulmonary embolism has reinforced our confidence in the accuracy and sensitivity of the technique. Our patients with an exacerbation of chronic obstructive lung disease differed from the patients in the earlier study in that no clinical signs suggesting deep venous thrombosis were observed. Nevertheless, similar collections of platelets were seen in those patients with thrombosis in all groups of patients. A negative scan was obtained from a patient two weeks before iliofemoral venous thrombosis and pulmonary emboli were found at postmortem examination; our experience with the indiumlabelled platelet technique leads us to believe that thrombosis occurred in the interval between the

Details of patients with and without thrombosis detected by the indium-labelled platelet technique

\begin{tabular}{|c|c|c|c|c|c|c|}
\hline & \multicolumn{3}{|c|}{$\begin{array}{l}\text { Patients with thrombosis } \\
(n=13)\end{array}$} & \multicolumn{3}{|c|}{$\begin{array}{l}\text { Patients without thrombosis } \\
(n=16)\end{array}$} \\
\hline & Mean & $S D$ & Range & Mean & $S D$ & Range \\
\hline 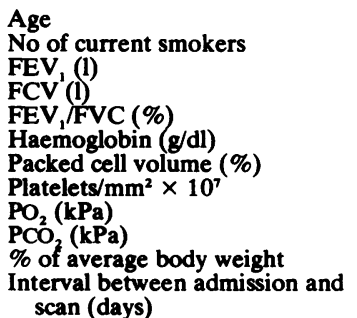 & $\begin{array}{l}71 \cdot 3 \\
1 \\
0 \cdot 72 \\
1 \cdot 52 \\
47 \\
14 \cdot 1 \\
45 \cdot 2 \\
276 \\
7 \cdot 88 \\
5 \cdot 93 \\
90 \cdot 4 \\
\\
8 \cdot 1\end{array}$ & $\begin{array}{c}8 \cdot 3 \\
\\
0 \cdot 40 \\
0 \cdot 62 \\
14 \\
2.0 \\
5 \cdot 4 \\
77 \\
1.62 \\
1 \cdot 16 \\
21 \cdot 2\end{array}$ & $\begin{array}{c}54-84 \\
\\
0 \cdot 25-1 \cdot 4 \\
0 \cdot 6-2 \cdot 7 \\
22-66 \\
12 \cdot 3-18 \cdot 9 \\
38-56 \\
151-369 \\
4 \cdot 1-10 \cdot 9 \\
4-8 \cdot 6 \\
64-124\end{array}$ & $\begin{array}{c}63.9 \\
3 \\
0.63 \\
1.44 \\
44.5 \\
14.4 \\
44.9 \\
301 \\
6.91 \\
6.05 \\
82.0\end{array}$ & $\begin{array}{c}11 \cdot 9 \\
\\
0.27 \\
0.42 \\
14.2 \\
1.4 \\
4 \cdot 6 \\
93 \\
1.26 \\
1.4 \\
14 \cdot 1\end{array}$ & $\begin{array}{c}36-81 \\
\\
0 \cdot 4-1 \cdot 5 \\
0 \cdot 8-2 \cdot 2 \\
25-59 \\
12 \cdot 2-17 \cdot 3 \\
37-55 \\
177-470 \\
4 \cdot 9-9 \cdot 3 \\
1 \cdot 7-8 \cdot 7 \\
58 \cdot 5-105\end{array}$ \\
\hline
\end{tabular}

$\mathrm{FEV}_{1}$ - forced expiratory volume in one second; $\mathrm{FVC}$ - forced vital capacity; $\mathrm{PO}_{2}, \mathrm{PCO}_{2}$ - oxygen and carbon dioxide tension. 
scan and death-although, as indicated above, false-negative scans probably occur occasionally. The wide variation in the interval between admission and scanning was a consequence of several factors. Platelet labelling was performed only when the patient was considered well enough to make the journey from the ward to the nuclear medicine department and to undergo the scanning procedure; thus the major factor determining the interval was the patient's speed of recovery from the exacerbation of chronic obstructive lung disease. Minor factors included the availability of indium and of scanning time. It might have been expected that deep venous thrombosis would occur more often in patients with more serious illness who were confined to bed for longer periods; but the interval between admission and scanning did not appear to influence its occurence (table). The period of immobility and illness before admission might have influenced the development of venous thrombosis but accurate data on this period are not available.

Our findings contrast with those of Prescott and colleagues, ${ }^{8}$ who found a low frequency of deep venous thrombosis in a group of North American patients with an exacerbation of chronic obstructive lung disease. Clearly major genetic and environmental differences must exist between a North American and a Scottish group of patients. In addition, the group studied by Prescott and colleagues had a lower mean age and was selected in that there had been no factors clearly precipitating the exacerbation of chronic obstructive lung disease. The criteria for admission to hospital are likely to differ between North America and Scotland; unfortunately the severity of disease in the two study populations cannot be compared from the available data. The major technique used for the diagnosis of deep venous thrombosis by Prescott and colleagues was that of ascending venography, which is noted for its accuracy in the calf but relative inaccuracy more proximally, particularly in the pelvis, ${ }^{9}$ where several of the thrombi in our patients were seen. Nevertheless, ascending venography and the indium-labelled platelet technique are both reliable methods for the diagnosis of distal thrombi and therefore the discrepancy between the findings of the two studies cannot be fully explained on the basis of the different diagnostic techniques used. Belch and her colleagues have recently observed that deep venous thrombosis, diagnosed by the iodine-131-labelled fibrinogen scanning technique, occurred in 13 of 50 (26\%) Scottish patients admitted to hospital with a chest infection or cardiac failure. ${ }^{10}$ There are several similarities between our patients and those of Belch and her colleagues, and their findings support our assertion that the incidence of deep venous throm- bosis in patients with an exacerbation of chronic obstructive lung disease is considerable. Their observations also suggest that by excluding patients in whom an infection was the clear cause of the exacerbation Prescott and colleagues may have excluded patients who would have been found to have deep venous thrombosis.

The results of our study do not indicate when thrombi occurred in relation to the exacerbation of respiratory symptoms, and thus do not imply that venous thrombosis and pulmonary embolism contributed to events leading to hospital admission, although this remains a possibility.

This study shows that deep venous thrombosis is common in patients admitted to hospital with exacerbations of chronic obstructive lung disease. In a high proportion of those with thrombosis the thrombus is present in veins proximal to the kneethat is, in areas in which thrombi are associated with a considerably greater risk of pulmonary embolism than are thrombi that are confined to veins below the knee. ${ }^{11}$

The diagnosis of pulmonary embolism is difficult in these patients, who already have abnormal pulmonary function and disturbed pulmonary perfusion and who may have pulmonary hypertension and heart failure. Furthermore, patients with severe chronic obstructive lung disease may tolerate pulmonary embolism badly. Our findings suggest that patients admitted with exacerbations may benefit from suitable prophylactic antithrombotic measures, such as low-dose subcutaneous heparin injections. ${ }^{10}$

We are grateful to Drs JAR Friend and JS Legge for their support and permission to study their patients and to Miss Christine Wallace for typing the manuscript.

\section{References}

' Baum GL, Fisher DF. The relationship of fatal pulmonary insufficiency with cor pulmonale, rightsided mural thrombi and pulmonary emboli: a preliminary report. Am J Med Sci 1960;240:609-12.

${ }^{2}$ Ryan SF. Pulmonary embolism and thrombosis in chronic obstructive emphysema. Am J Pathol 1963;43:767-73.

${ }^{3}$ Sharma GVRK, Sasahara AA. Diagnosis of pulmonary embolism in patients with chronic obstructive pulmonary diseases. J Chron Dis 1975;28:253-7.

4 Gibbs NM. Venous thrombosis of the lower limbs with particular reference to bed rest. $B r J$ Surg 1957;45:209-36.

${ }^{5}$ Havig O. Deep vein thrombosis and pulmonary emboli. Acta Chir Scand 1977;478,Suppl:42-7.

${ }^{6}$ Fenech A, Hussey J, Smith FW, Dendy PP, Bennett B, Douglas AS. Diagnosis of deep vein thrombosis using autologous indium-111-labelled platelets. $\mathrm{Br}$ Med $\mathrm{J}$ 1981;282:1020-2. 
' Diem K, Lentner C. Scientific tables. 7th ed. Basel: Geigy Pharmaceuticals, 1970.

${ }^{8}$ Prescott SM, Richards KL, Tikoff G, Armstrong JD, Shigeoka JW. Venous thromboembolism in decompensated chronic obstructive pulmonary disease. $\mathrm{Am}$ Rev Respir Dis 1981;123:32-6.

${ }^{9}$ Bettmann NA, Salzman EW. Recent advances in the diagnosis of deep vein thrombosis and pulmonary embolism. In: Poller L, ed. Recent advances in blood coagulation. Edinburgh: Churchill Livingstone, 1981: 287-317.

${ }^{10}$ Belch JJ, Lowe GDO, Ward AG, Forbes, CD, Prentice CRM. Prevention of deep vein thrombosis in medical patients by low-dose heparin. Scott Med J 1981;26:115-7.

" Moser KM, Lemoine JR. Is embolic risk conditioned by location of deep venous thrombosis? Ann Intern Med $1981 ; 94: 439-44$. 